\title{
Administration of Nivolumab in Metastatic Renal Cell Cancer Following Treatment With mTOR Inhibitors
}

\author{
JINDRICH KOPECKY ${ }^{1 *}$, ONDREJ KUBECEK ${ }^{1 *}$, TOMAS BUCHLER $^{2}$, BOHUSLAV MELICHAR $^{1,3}$, \\ ALEXANDR POPRACH ${ }^{4,5}$, MILADA ZEMANOVA ${ }^{6}$, JANA KATOLICKA $^{7}$, IGOR KISS $^{4,5}$, \\ JAROSLAV HAJEK $^{8}$, HANA STUDENTOVA ${ }^{3}$ and MARTINA SPISAROVA ${ }^{3}$ \\ ${ }^{1}$ Department of Oncology and Radiotherapy Faculty of Medicine and \\ University Hospital Hradec Kralove, Hradec Kralove, Czech Republic; \\ ${ }^{2}$ Department of Oncology, First Faculty of Medicine, \\ Charles University and Thomayer Hospital, Prague, Czech Republic; \\ ${ }^{3}$ Department of Oncology, Palacky University Medical School and Teaching Hospital, Olomouc, Czech Republic; \\ ${ }^{4}$ Department of Comprehensive Cancer Care, Masaryk Memorial Cancer Institute, Brno, Czech Republic; \\ ${ }^{5}$ Department of Comprehensive Cancer Care, Faculty of Medicine, Masaryk University, Brno, Czech Republic; \\ ${ }^{6}$ Department of Oncology, First Faculty of Medicine Charles University \\ and General University Hospital, Prague, Czech Republic; \\ ${ }^{7}$ Department of Oncology, St. Anne's University Hospital Brno, Brno, Czech Republic; \\ ${ }^{8}$ Department of Oncology, University Hospital Ostrava, Ostrava, Czech Republic
}

\begin{abstract}
Background/Aim: Immunotherapy with checkpoint inhibitors is currently considered a cornerstone of metastatic renal clear cell cancer (mRCC) therapy. Despite the general improvement in the survival of patients with $m R C C$, there are some clinical situations that have not been specifically evaluated in clinical trials, such as the use of everolimus before nivolumab. Patients and Methods: We performed a retrospective analysis evaluating the efficacy of nivolumab in the real-world setting, including a subset of patients with previous mTOR inhibitor therapy. Results: From a total of 56 patients, 25 were pre-treated with everolimus before receiving nivolumab. The overall progression-free survival (PFS), overall survival (OS), and objective response rate were 10.3, 21.3 months, and $34 \%$, respectively. There were no statistically significant differences in patients who were or were not pretreated with everolimus. Conclusion: $m R C C$ patients should
\end{abstract}

This article is freely accessible online.

\footnotetext{
*These Authors contributed equally to this study.

Correspondence to: Jindrich Kopecký, Department of Clinical Oncology and Radiotherapy, University Hospital Hradec Kralove, Sokolská 581, Hradec Kralove 50005, Czech Republic. Tel: +42 0495832176, e-mail: jindrich.kopecky@fnhk.cz
}

Key Words: Renal cell cancer, nivolumab, mTOR inhibitor, neutrophil-lymphocyte ratio, platelet-lymphocyte ratio, systematic inflammation index. be treated with checkpoint inhibitors and prior use of mTOR inhibitors should not be a definitive exclusion criterium.

Significant progress has been made in the treatment of metastatic renal cell carcinoma (mRCC) during the last decades. Currently available drugs for systemic therapy include anti-VEGF multikinase inhibitors (sunitinib, sorafenib, pazopanib, tivozanib or axitinib), cytokines (interferon- $\alpha$ and interleukin-2), anti-VEGF antibody (bevacizumab), and mTOR inhibitors (temsirolimus and everolimus). This armamentarium has been recently expanded by cabozantinib, lenvatinib, and the immune checkpoint inhibitors (CPI) nivolumab, ipilimumab, pembrolizumab, and avelumab.

Immunotherapy with $\mathrm{CPI}$ is currently considered a cornerstone of mRCC front-line therapy. In addition, nivolumab, an anti-PD-1 antibody, has proven its efficacy in the second- and third-line therapy of $\mathrm{mRCC}$ in a randomised phase III trial, CheckMate 025 (1).

Despite the general improvement in survival of patients with $\mathrm{mRCC}$ treated with CPI, there is notable variability in therapeutic response, which is currently impossible to predict. This is a burning question, and a big effort has been made in search for predictive biomarkers that would predict patient benefit, risk of toxicities, and guide combination approaches.

The currently used therapeutic approach in mRCC consists of sequential or combination administration of active agents. However, the data of large prospective trials usually do not address the question of optimal treatment sequence, and few 
prospective trials have provided a head-to-head comparison of treatment sequences. An example is the use of nivolumab in patients who were previously treated with an mTOR inhibitor (temsirolimus or everolimus). Although the management of this clinical scenario has never been specifically evaluated in prospective trials, a course of immunotherapy is generally advised as a part of treatment sequence in mRCC patients. Registry-based retrospective studies can provide valuable information on various treatment sequences that could not have been prospectively evaluated in clinical trials. A retrospective analysis evaluating the efficacy of CPI in patients with previous mTOR inhibitor therapy was carried out using data from the nivolumab Early Access Program (EAP) in the Czech Republic.

The primary aim of this study was to assess the safety and efficacy of nivolumab used in the real-world setting and to assess the efficacy of nivolumab administered following treatment with an mTOR inhibitor. The secondary aim was to evaluate potential clinical or laboratory biomarkers that could be used to predict therapeutic outcome.

\section{Patients and Methods}

Study population. The study included patients enrolled in the Early Access Program (EAP) of nivolumab therapy in mRCC between November 2015 and June 2016. The EAP allowed to enroll patients with documented progressive disease following the current standard-of-care therapy for mRCC. Before enrollment, all patients had signed a written informed consent. The study was conducted according to the guidelines of the Declaration of Helsinki.

The inclusion criteria were specified as histologically confirmed RCC with a clear cell component, and at least one line of prior antiangiogenic therapy in the advanced or metastatic disease setting. Prior cytokine (e.g., interferon- $\alpha$ ), vaccine, or cytotoxic drug therapy was also allowed.

Therapy. The patients received $3 \mathrm{mg} / \mathrm{kg}$ nivolumab once every two weeks. Treatment was to continue until progressive disease or unacceptable toxicity for a maximum of two years of therapy.

All patient data were obtained from the patient clinical files and laboratory reports. Regular safety assessments were performed during the treatment period, including periodic physical evaluation and laboratory examination (peripheral blood cell count, renal and hepatic function, pancreatic enzymes, and thyroid function). Data were collected centrally and included the date of primary tumor diagnosis, the date of diagnosis of metastatic disease, the sites of metastases, International Metastatic RCC Database Consortium risk score (IMDC), dates of each therapeutic sequence, adverse events including grade, baseline laboratory findings before the start of immunotherapy including blood count, neutrophils, lymphocytes, C-reactive protein (CRP), serum calcium, lactate dehydrogenase, and albumin, Eastern Cooperative Oncology Group performance status (ECOG) performance status, weight and height, date of the best response to immunotherapy, type of the best response, either complete response (CR), partial response (PR), stable disease (SD), or progressive disease (PD), date of last control or death, date of progression, type of nephrectomy (radical/cytoreductive/none) and information whether radiation therapy was performed. Based on initial blood count parameters, peripheral blood cell count-derived ratios, such as platelet-lymphocyte-ratio (PLR), neutrophillymphocyte-ratio (NLR), lymphocyte-monocyte-ratio (LMR), and systemic immune-inflammation index (SII) were calculated.

Endpoints and response evaluation. A radiographic CT assessment was performed before treatment initiation, then every twelve to sixteen weeks and within 6-8 weeks after the first CT documenting PD to confirm the initial assessment of disease progression.

Response to therapy was evaluated using the Response Evaluation Criteria in Solid Tumors 1.1 (RECIST). Progression-free survival (PFS) and/or objective response were used as primary endpoints to evaluate the treatment efficacy and overall response rate (ORR). According to the best documented response, patients were classified as responders or non-responders. Best responder was the patient who achieved CR or PR and non-responder was the patient with PD. In the case of first documented disease progression, patients were allowed to continue with nivolumab at the discretion of attending physician.

PFS was calculated from the start of nivolumab administration until disease progression or death. Overall survival (OS) on nivolumab was calculated from the beginning of nivolumab treatment until death. The cut-off date was August 1, 2019. Patients who were alive or without documented disease progression were censored at the date of the last follow-up visit.

Further, we defined the disease control rate (DCR) as a sum of CR, $\mathrm{PR}$, and SD and treatment-free survival (TFS) as the time interval between the end of nivolumab therapy to the beginning of subsequent systemic therapy or death. Patients alive without subsequent therapy were censored at the date of the last follow-up visit.

Statistics. Data are summarized as frequencies for categorical variables and median and range values for continuous variables. Multivariable logistic regression analysis was used to analyze the association between the overall response and a set of independent explanatory variables. The Cox proportional hazards regression was used to estimate hazard ratios and their $95 \%$ confidence intervals for OS and PFS. The forward selection method of explanatory variables was used. The maximum number of the estimated parameters was pre-defined as the largest integer less than or equal to the number of observed events divided by 5 to guard against over-parametrization.

The set of the explanatory variables included mTOR inhibitor administration as a factor indicating whether or not an inhibitor mTOR (everolimus) was used before the start of patient participation, the age at the start of EAP, the time between the diagnosis of metastatic disease and the inclusion into the EAP, the line of therapy, IMDC (2), ECOG performance status, metastatic sites including lung, liver, nodes, bones and brain, previous radiotherapy, treatment-related toxicity and the SII index (3), calculated by the following formula: platelet count $\times$ neutrophil count/lymphocyte count. The estimates of the models were given as hazard ratios (HR) for Cox model and odds ratios (OR) for logistic regression, with $95 \%$ confidence intervals $(\mathrm{CI})$.

Survival (PFS and OS) was analyzed by the Kaplan-Meier method and subgroups were compared using the log-rank test. All $p$-values were calculated using two-sided tests, and values $<0.05$ were considered statistically significant. Statistical analyses were conducted using S+ ${ }^{\circledR} 8.2$ (TIBCO Software Inc., Palo Alto, CA, USA) and/or NCSS 2020 (NCSS, LLC. Kaysville, UT, USA). 
Table I. Baseline characteristics.

\begin{tabular}{|c|c|c|c|c|c|c|c|}
\hline \multirow[b]{2}{*}{ Gender } & \multicolumn{2}{|c|}{$\begin{array}{l}\text { All patients } \\
\mathrm{n}=56\end{array}$} & \multicolumn{2}{|c|}{$\begin{array}{l}\text { With previous everolimus } \\
\qquad \mathrm{n}=25\end{array}$} & \multicolumn{2}{|c|}{$\begin{array}{l}\text { Without previous everolimus } \\
\qquad \mathrm{n}=31\end{array}$} & \multirow{2}{*}{$\begin{array}{c}p \text {-Value } \\
0.38\end{array}$} \\
\hline & $\mathrm{N}$ & $\%$ & $\mathrm{~N}$ & $\%$ & $\mathrm{~N}$ & $\%$ & \\
\hline Male & 44 & $(79 \%)$ & 21 & $(84 \%)$ & 23 & $(74 \%)$ & \\
\hline Female & 12 & $(21 \%)$ & 4 & $(16 \%)$ & 8 & $(26 \%)$ & \\
\hline Nephrectomy & & & & & & & 0.50 \\
\hline None & 5 & $(9 \%)$ & 3 & $(12 \%)$ & 2 & $(7 \%)$ & \\
\hline Radical & 41 & $(73 \%)$ & 19 & $(76 \%)$ & 22 & $(70 \%)$ & \\
\hline Cytoreductive & 10 & $(18 \%)$ & 3 & $(12 \%)$ & 7 & $(23 \%)$ & \\
\hline Metastasis site & & & & & & & 0.32 \\
\hline Lung & 39 & $(70 \%)$ & 20 & $(80 \%)$ & 19 & $(61 \%)$ & \\
\hline Liver & 9 & $(16 \%)$ & 5 & $(20 \%)$ & 4 & $(13 \%)$ & \\
\hline Lymph node & 22 & $(39 \%)$ & 10 & $(40 \%)$ & 12 & $(39 \%)$ & \\
\hline Bone & 31 & $(55 \%)$ & 13 & $(52 \%)$ & 18 & $(58 \%)$ & \\
\hline Brain & 7 & $(13 \%)$ & 6 & $(24 \%)$ & 1 & $(3 \%)$ & \\
\hline Radiotherapy & & & & & & & 0.93 \\
\hline Not used & 25 & $(45 \%)$ & 11 & $(44 \%)$ & 14 & $(45 \%)$ & \\
\hline Used & 31 & $(55 \%)$ & 14 & $(56 \%)$ & 17 & $(55 \%)$ & \\
\hline ECOG (WHO) & & & & & & & 0.51 \\
\hline 0 & 15 & $(27 \%)$ & 5 & $(20 \%)$ & 10 & $(32 \%)$ & \\
\hline 1 & 36 & $(64 \%)$ & 17 & $(68 \%)$ & 19 & $(61 \%)$ & \\
\hline 2 & 5 & $(9 \%)$ & 3 & $(12 \%)$ & 2 & $(7 \%)$ & \\
\hline IMDC (Heng) & & & & & & & 0.49 \\
\hline Good & 20 & $(36 \%)$ & 7 & $(28 \%)$ & 13 & $(42 \%)$ & \\
\hline Intermediate & 31 & $(55 \%)$ & 15 & $(60 \%)$ & 16 & $(52 \%)$ & \\
\hline Poor & 5 & $(9 \%)$ & 3 & $(12 \%)$ & 2 & $(6 \%)$ & \\
\hline Nivolumab line & & & & & & & $<0.0001$ \\
\hline 2 & 17 & $(30 \%)$ & 0 & $(0 \%)$ & 17 & $(55 \%)$ & \\
\hline 3 & 19 & $(34 \%)$ & 6 & $(24 \%)$ & 13 & $(42 \%)$ & \\
\hline 4 & 12 & $(21 \%)$ & 11 & $(44 \%)$ & 1 & $(3 \%)$ & \\
\hline 5 & 4 & $(7 \%)$ & 4 & $(16 \%)$ & 0 & $(0 \%)$ & \\
\hline 6 & 4 & $(7 \%)$ & 4 & $(16 \%)$ & 0 & $(0 \%)$ & \\
\hline
\end{tabular}

ECOG: Eastern Cooperative Oncology Group performance status; IMDC: International Metastatic RCC Database Consortium.

\section{Results}

Patients. A total of 56 patients were enrolled in the EAP between November 2015 and June 2016. The median followup was 21.3 months (range=0.6-42.4 months). The majority of patients were males $(79 \%)$. The median age of the patients at the time of EAP enrolment was 66.5 years (range=44.379.3 years). Twenty-five patients $(44.6 \%)$ were pre-treated with everolimus before receiving a CPI. The baseline characteristics are listed in Table I.

At the time of this analysis (August 1, 2019), 16 patients $(28.6 \%)$ were still alive without continuing treatment with nivolumab. The median duration of treatment was 9.7 months (range=0.4-38.7 months), and the median number of administered doses was 21 (range $=1-76$ ); the course of a 2year therapy was completed by 15 patients $(27 \%)$. A total of 31 patients had undergone radiotherapy before or during EAP. Among the 41 patients (73\%), 36 patients (88\%), 3 patients $(7 \%)$ and 2 patients (5\%) discontinued treatment because of disease progression, serious adverse events (AEs) and death, respectively. There were 14 patients $(26 \%)$ treated beyond the first documented progression, including 9 patients (16\%) in whom subsequent response was observed (the initial progression was therefore considered as pseudo-progression).

The PFS and OS for all patients were 10.3 months [confidence interval $(\mathrm{CI})=95 \% ; 5.29-20.83)]$ and 21.3 months (95\% CI=15.05-29.73), respectively (Figure 1A and B). The objective response rate (ORR) for all patients was $33.9 \%$, and the disease control rate (DCR) $66 \%$. The duration of response (DOR) for all patients with DCR was 15.1 months (95\% CI=7.56-35.55). The one-year percentage of TFS was $54 \%$. The ongoing responses are depicted in Figure 2.

In a univariable analysis the presence of liver metastases was identified as negative prognostic factor for PFS $(\mathrm{HR}=3.42 ; 95 \% \mathrm{CI}=1.54-7.57, p=0.002)$ and an inferior performance status was negative prognostic factor for OS $(\mathrm{HR}=2.29 ; 95 \% \mathrm{CI}=1.05-5.02, p=0.038)$. In a multivariable analysis, the presence of liver metastases was a significant 
A

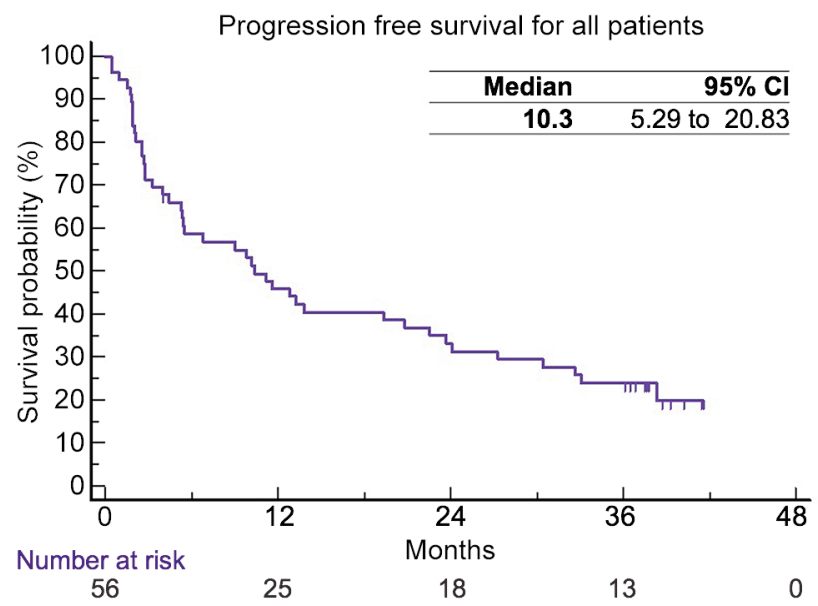

B

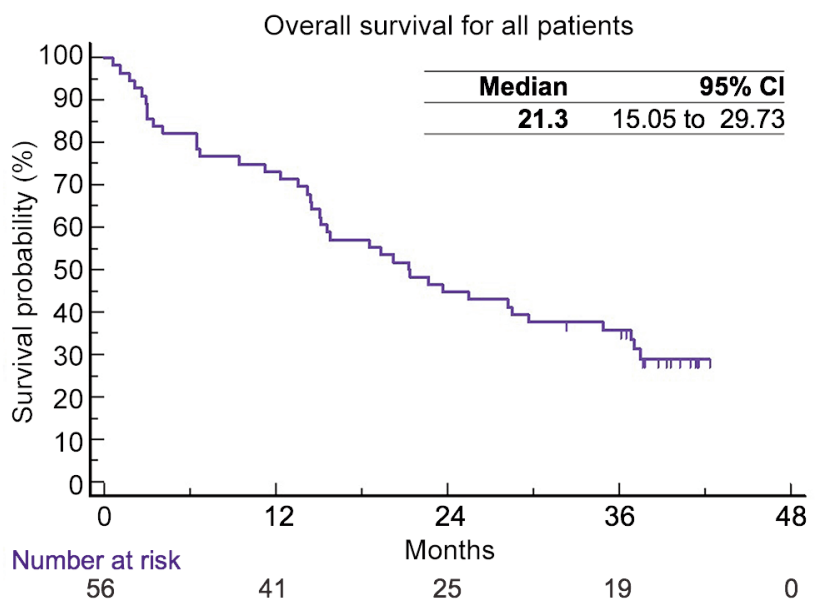

Figure 1. Survival analysis using the Kaplan-Meier method for all patients: (A) Progression-free survival; (B) Overall survival.

negative prognostic factor for PFS $(\mathrm{HR}=3.60 ; 95 \% \mathrm{CI}=1.44-$ $9.00, p=0.006)$, while the presence of bone metastases and the number of previous therapy lines (three versus two) were associated with improved PFS $(\mathrm{HR}=0.36 ; 95 \% \mathrm{CI}=0.17-0.76$, $p=0.008$, and $\mathrm{HR}=0.40 ; 95 \% \mathrm{CI}=0.17-0.95, p=0.037)$, respectively. Other negative prognostic factors for OS identified in the multivariable analysis were inferior performance status and the presence of lung metastases $(\mathrm{HR}=2.58 ; 95 \% \mathrm{CI}=1.13-5.89, p=0.025$, and $\mathrm{HR}=2.42$; $95 \% \mathrm{CI}=1.06-5.53, p=0.036)$. Age $>65$ years was the only independent predictive factor for ORR in a multivariable analysis $(\mathrm{HR}=0.29 ; 95 \% \mathrm{CI}=0.09-0.93, p=0.038)$.

Nivolumab efficacy in patients pre-treated with everolimus. Before entering nivolumab EAP program, 25 (45\%) patients were pre-treated with everolimus, whereas 31 (55\%) patients were everolimus naïve (Table I). There were no statistically significant differences between the groups, except for the frequency in what the therapeutic line nivolumab was used, which was also why the time between diagnosis of metastatic disease and the start of EAP was significantly longer in patients with previous everolimus (47.4 vs. 28.7 months, $p=0.014$ ).

The PFS and OS for both groups were not statistically different, however, the median values were better in patients without prior everolimus therapy (median $13.8 \mathrm{vs} .6 .7$ and 25.5 vs. 15.1 months for PFS and OS, respectively) (Figure $3 \mathrm{~A}$ and $\mathrm{B})$. Objective responses were more numerically common in patients without previous everolimus therapy (CR $6.4 \%$ and $4 \%$, and PR $35.5 \%$ and $20 \%$, respectively). The median of DOR for nivolumab was not affected by previous treatment with everolimus (14.0 versus 16.3 months, $p=0.242$; Figure 2). Long-term responders were observed among patients who had prior everolimus.
Neutrophil-lymphocyte ratio (NLR), platelet-lymphocyte ratio (PLR), systemic immune-inflammation index (SII). The possible prognostic impact of baseline NLR, PLR, and SII values was analysed. Because of the unknown optimal cutoff value for NLR, PLR, and SII, cut-off values were determined based on literature search (4-6). PFS and OS were inferior in patients with NLR ratio equal or above the cut-off of $5(\mathrm{HR}=2.22 ; 95 \% \mathrm{CI}=0.96-5.15 ; p=0.06$, and $\mathrm{HR}=2.99 ; 95 \% \mathrm{CI}=1.27-7.06 ; p=0.01$, respectively). In the case of PLR ratios, there was statistical trend for worse PFS in patients with values equal or above 300 ( $\mathrm{HR}=2.46$; $95 \% \mathrm{CI}=0.96-6.29, p=0.06)$. PLR values equal or above 300 were associated with inferior $\mathrm{OS}(\mathrm{HR}=4.11 ; 95 \% \mathrm{CI}=1.52$ $11.07, p=0.005)$. SII values equal or above 750 were associated with statistically significant inferior PFS (median 13.8 vs. 4.0 months, $\mathrm{HR}=1.93 ; 95 \% \mathrm{CI}=1.03-3.66 ; p=0.04)$, and $\mathrm{OS}$ (median 29.7 vs. 14.5 months, $\mathrm{HR}=2.11$; $95 \% \mathrm{CI}=1.09-4.06 ; p=0.02)$.

Safety analysis. Only treatment-related AEs were recorded during the EAP. No unexpected treatment-related AEs were observed. The list of AEs is shown in Table II. There was one case of treatment-related death from encephalopathy, which has been reported as a case study earlier (7). Treatment-related grade 3-4 AEs occurred in 4 patients $(7.1 \%)$ and ultimately lead to treatment cessation in three of these patients.

Responders and non-responders. In the present cohort of patients, 19 patients with best observed response corresponding to $\mathrm{CR}$ (3 patients) and PR (16 patients) were considered as responders. Patients with first response evaluated as PD were considered as non-responders (18 


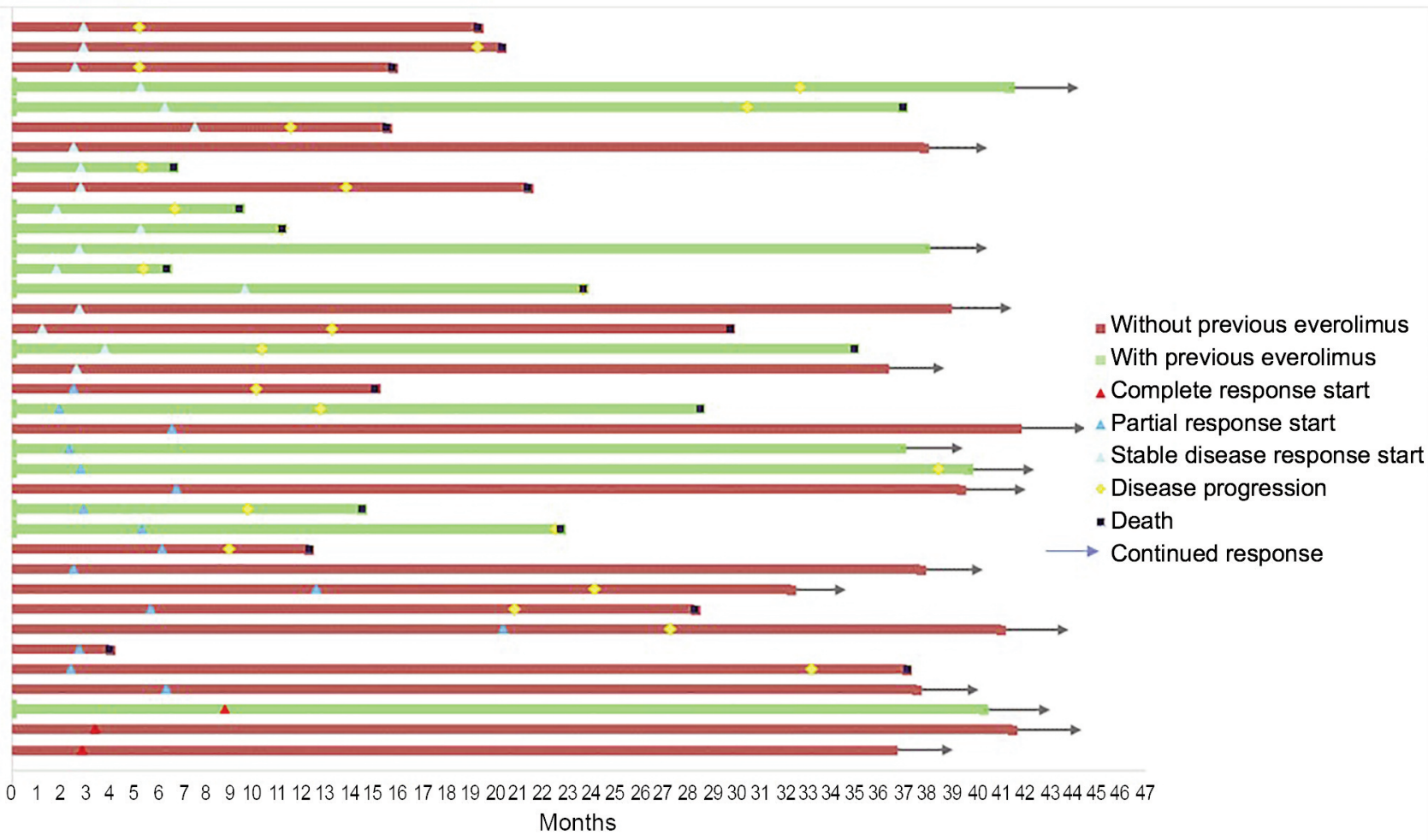

\begin{tabular}{rrr}
\hline DOR & Media $(95 \% \mathrm{Cl})$ & P; HR (95\% Cl) \\
\hline All patients $(n=37)$ & $15.1(7.56$ to 35.55$)$ & \\
With previous everolimus $(n=15)$ & $16.3(7.56$ to 30.69$)$ & $p=0.24 ; 1.64(0.72$ to 3.74$)$ \\
Without previous everolimus $(n=22)$ & $14.0(4.90$ to 27.30$)$ & \\
\hline
\end{tabular}

Figure 2. Swimmer plot for patients with complete response (CR), partial response (PR), and stable disease (SD). DOR: Duration of response.

patients). One patient who could not be radiological evaluated as well as patients with stable disease were excluded from the analysis. Univariable and multivariable logistic regression analyses were performed to assess the association between the overall response and a set of independent explanatory variables. From all variables, only two factors showing association with the response could be identified. In a multivariable analysis, the presence of liver metastases (inferior response) and the presence of treatment-related toxicity (improved response) showed statistically significant association with response $(\mathrm{OR}=0.01 ; 95 \% \mathrm{CI}=0.0-0.31$, $p=0.009$, and $\mathrm{OR}=22.73 ; 95 \% \mathrm{CI}=1.49-345.97, p=0.025$; respectively).

Fast progressors and CPI. Among the 56 patients treated with nivolumab in mRCC EAP, 17 patients showed rapid progression while treated with the first-line therapy. Among these patients, 8 patients received nivolumab as second-line therapy whereas the remaining patients were treated with TKI (6 patients) or mTOR ( 3 patients). The majority of patients received sunitinib as the first-line therapy. Median PFS for patients with nivolumab in the second line was 11.6 months (95\% CI=7.9-15.2), and the best objective response was PR in $50 \%$ (4 patients), and the DCR in this group of patients was $87.5 \%$ (7 patients).

\section{Discussion}

The present retrospective study evaluated the safety and efficacy of nivolumab in patients with $\mathrm{mRCC}$ treated in the EAP program in the Czech Republic, focusing on the clinical scenario of everolimus used before nivolumab. 

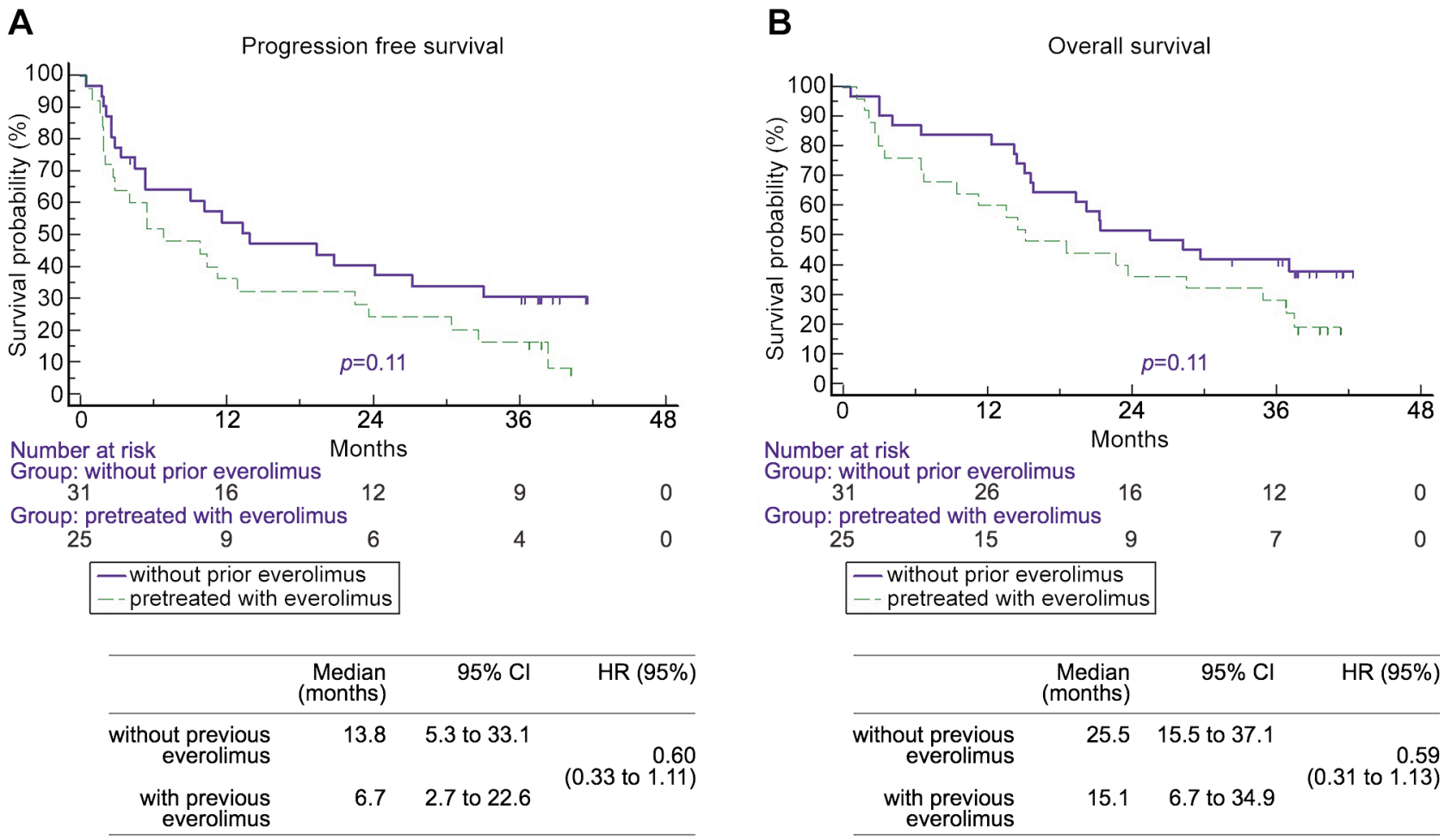

Figure 3. Survival analysis for patients with and without previous everolimus treatment: (A) Progression-free survival; (B) Overall survival.

In the present real-life analysis, nivolumab therapy proved to be safe and tolerable, with only $7.3 \%$ of patients discontinuing treatment because of severe AEs. However, two patients died during the EAP, including one patient who died of immunotherapy-related neurotoxicity (7). The lower incidence of AEs could, however, be explained by possible underreporting side effects within EAP, in contrast to monitored prospective clinical trials.

The other three real-world studies (the French Nivoren study, the Italian EAP and NORA studies) analysed similar patient populations as the present study (8-11). The results from Nivoren and the Italian EAP and NORA studies are as follows: for ORR 20.8, 23.1 and 27\%, PFS 3.2 and 4.4 months (for NORA study not reported), OS 24.5 months in Nivoren trial. The OS for the whole study population of the Italian EAP has not been reported. However, the OS, reported separately for patients with and without AEs, was 22.5 and 16.4 months, respectively. The reported OS from the NORA study was 26.9 months.

Although it is not possible to directly compare the results of these studies and the Checkmate 025 study, all of the studies reported similar median OS of approximately 25 months. However, there is a difference in ORR and PFS results between the present analysis and other studies. We noted ORR of $33 \%$ and PFS of 10.5 months whereas for other studies, including Checkmate 025, the ORR was $25 \%$ and PFS approximately 4 months.

The better results of ORR and PFS in the present study might be explained partially by a patient selection resulting in higher and lower percentages of patients in the good and poor prognostic group, respectively. The fact that more than half of the patients had received radiotherapy could represent another explanation. In most cases, radiotherapy was aimed at bone metastases $(70 \%)$. The use of radiotherapy was not assessed in the other real-world studies mentioned above. Because of the retrospective design, we were not able to analyse whether the abscopal effect of radiotherapy could have an effect. However, it is possible that the use of radiotherapy might have a synergistic effect with immunotherapy resulting in improved therapeutic outcomes.

Another simple explanation for better results in ORR and PFS is the non-randomized study design. A factor that could also be responsible for differences in PFS and ORR might be the administration of everolimus before nivolumab. We hypothesize that there is no difference based on whether the patient is or is not pre-treated with everolimus. Although we did not find any statistically significant difference between patients with and without everolimus, we observed numerically better results for patients who were not pre-treated with everolimus. It should be mentioned that the cohort of patients 
Table II. Overview of adverse events during nivolumab therapy.

\begin{tabular}{|c|c|c|c|c|c|c|}
\hline \multirow[t]{2}{*}{ System organ class } & \multicolumn{6}{|c|}{ Toxicity grade } \\
\hline & 1 & 2 & 3 & 4 & 5 & Total \\
\hline \multicolumn{7}{|l|}{ Endocrine, N (\%) } \\
\hline Thyreopathy & $2(3.6 \%)$ & $0(0.0 \%)$ & $1(1.8 \%)$ & $0(0.0 \%)$ & $0(0.0 \%)$ & $3(5.4 \%)$ \\
\hline \multicolumn{7}{|l|}{ General N (\%) } \\
\hline Fatigue & $5(8.9 \%)$ & $4(7.1 \%)$ & $0(0.0 \%)$ & $0(0.0 \%)$ & $0(0.0 \%)$ & $9(16.0 \%)$ \\
\hline Infusion reaction & $0(0.0 \%)$ & $1(1.8 \%)$ & $0(0.0 \%)$ & $0(0.0 \%)$ & $0(0.0 \%)$ & $1(1.8 \%)$ \\
\hline \multicolumn{7}{|l|}{ Gastrointestinal N (\%) } \\
\hline Obstipation & $1(1.8 \%)$ & $0(0.0 \%)$ & $0(0.0 \%)$ & $0(0.0 \%)$ & $0(0.0 \%)$ & $1(1.8 \%)$ \\
\hline Diarrhoea & $2(3.6 \%)$ & $1(1.8 \%)$ & $1(1.8 \%)$ & $0(0.0 \%)$ & $0(0.0 \%)$ & $4(7.1 \%)$ \\
\hline \multicolumn{7}{|l|}{ Neurological N (\%) } \\
\hline Encephalitis & $0(0.0 \%)$ & $0(0.0 \%)$ & $0(0.0 \%)$ & $0(0.0 \%)$ & $1(1.8 \%)$ & $1(1.8 \%)$ \\
\hline \multicolumn{7}{|l|}{ Skin N (\%) } \\
\hline Any AR & $6(10.7 \%)$ & $1(1.8 \%)$ & $0(0.0 \%)$ & $0(0.0 \%)$ & $0(0.0 \%)$ & $7(12.5 \%)$ \\
\hline \multicolumn{7}{|l|}{ Respiratory N (\%) } \\
\hline Cough & $1(1.8 \%)$ & $0(0.0 \%)$ & $0(0.0 \%)$ & $0(0.0 \%)$ & $0(0.0 \%)$ & $1(1.8 \%)$ \\
\hline Pneumonitis & $1(1.8 \%)$ & $1(1.8 \%)$ & $1(1.8 \%)$ & $0(0.0 \%)$ & $0(0.0 \%)$ & $3(5.4 \%)$ \\
\hline \multicolumn{7}{|l|}{ Muscular N (\%) } \\
\hline Arthralgia & $2(3.6 \%)$ & $2(3.6 \%)$ & $0(0.0 \%)$ & $0(0.0 \%)$ & $0(0.0 \%)$ & $4(7.1 \%)$ \\
\hline \multicolumn{7}{|l|}{ Hepatic N (\%) } \\
\hline Elevation of liver enzymes & $2(3.6 \%)$ & $0(0.0 \%)$ & $0(0.0 \%)$ & $0(0.0 \%)$ & $0(0.0 \%)$ & $2(3.6 \%)$ \\
\hline \multicolumn{7}{|l|}{ Cardiovascular N (\%) } \\
\hline Cardiotoxicity & $0(0.0 \%)$ & $0(0.0 \%)$ & $1(1.8 \%)$ & $0(0.0 \%)$ & $0(0.0 \%)$ & $1(1.8 \%)$ \\
\hline \multicolumn{7}{|c|}{ Blood and metabolic disorders $\mathrm{N}(\%)$} \\
\hline Anaemia & $0(0.0 \%)$ & $1(1.8 \%)$ & $0(0.0 \%)$ & $0(0.0 \%)$ & $0(0.0 \%)$ & $1(1.8 \%)$ \\
\hline Mineral imbalance & $0(0.0 \%)$ & $1(1.8 \%)$ & $0(0.0 \%)$ & $0(0.0 \%)$ & $0(0.0 \%)$ & $1(1.8 \%)$ \\
\hline Hyperlipidaemia & $1(1.8 \%)$ & $0(0.0 \%)$ & $0(0.0 \%)$ & $0(0.0 \%)$ & $0(0.0 \%)$ & $1(1.8 \%)$ \\
\hline Hyperglycaemia & $1(1.8 \%)$ & $0(0.0 \%)$ & $0(0.0 \%)$ & $0(0.0 \%)$ & $0(0.0 \%)$ & $1(1.8 \%)$ \\
\hline
\end{tabular}

pre-treated with everolimus received more therapy lines before enrollment and could therefore be long-term survivors with more favorable tumor biology and lower tumor aggressiveness. This might, together with a limited sample size, lead to nonsignificant differences in outcome based on previous everolimus therapy. Two other real-world studies (the Italian EAP and NIVOREN) analysed the impact of everolimus on nivolumab efficacy. In the preliminary exploratory analysis of the NIVOREN study, the authors showed a borderline, but statistically significant prognostic impact of previous everolimus $(\mathrm{HR}=1.39 ; 95 \% \mathrm{CI}=1.01-1.92, p=0.044)(8,9)$, whereas in the Italian EAP, prior everolimus therapy showed just a statistically non-significant trend for inferior OS $(\mathrm{HR}=1.30 ; 95 \% \mathrm{CI}=0.95-1.76, p=0.10)(10)$. In the NIVOREN study, there was a lower proportion of patients treated with everolimus (22\%) and the reported HR derived from preliminary analysis with a median follow-up of 13.1 months. In the Italian EAP, the follow-up was 11.9 months, and in both studies, there were still patients on therapy (15\% in the NIVOREN study and $28.3 \%$ in the Italian EAP). In the present study, the small patient number is a limitation, which might be responsible for the non-statistically significant differences.
Until recently, the strategy for the second-line therapy was based on a switch to either a different TKI $(12,13)$, or an mTOR inhibitor (14) following disease progression on the first-line TKI therapy. The situation has become more complicated with the advent of a more potent TKI cabozantinib (15), nivolumab (1), and the combination of everolimus with lenvatinib (16).

The use of nivolumab in the second and further lines of mRCC therapy is based on the results of the Checkmate 025 trial. As everolimus was used as the active comparator in the study, patients were not allowed to be pre-treated with this mTOR inhibitor (1). Despite the fact that everolimus is currently not preferred over nivolumab for the second or third line, the advent of lenvatinib combined with everolimus (16), makes this issue again clinically relevant. Following the results from METEOR and Checkmate 025 clinical trials $(1,15)$, the use of everolimus prior to nivolumab is considered as obsolete. There seems to be also a scientific rationale to avoid using everolimus before nivolumab. The use of everolimus is associated with immunological alterations in circulating immune cells (increase of Tregs, monocytic myeloid-derived suppressor 
cells, and decrease NK cells and dendritic cells) and increase PD-L1 expression in $\operatorname{RCC}(17,18)$. On the other hand, with the advent of a new combination everolimus plus lenvatinib, there is a new potential for using of everolimus before nivolumab. This clinical scenario needs to be further examined. Based on previous reports, the efficacy of lenvatinib against cancer relies not only on its antiangiogenic activity and its ability to suppress tumor proliferation, but it is also associated with enhanced tumor infiltration and activation of NK cells (19). In the light of these theories, the results of the Italian EAP as well as the present analysis provide encouraging data on the efficacy of sequential strategy involving everolimus and nivolumab.

Peripheral blood cell count-derived ratios such as NLR or PLR are simple and easily determined biomarkers that can be obtained from routine peripheral blood testing. It has been previously reported that higher values of these parameters are predictive of worst OS and PFS (20-22). A newer derived parameter obtained from peripheral blood is the SII. As compared to NLR and PLR, this parameter represents a more robust assessment of the balance between an inflammatory condition and host immune responses, combining the information contained in NLR and PLR indices (4). To the best of our knowledge, the present study is the first that evaluated all these three parameters with regard to OS and PFS in this setting. In the present cohort of patients, we found NLR, PLR and SII parameters to be promising biomarkers associated with the response.

Despite all progress in mRCC therapy, there is still a rather high proportion of patients, between 20 to $30 \%$, with a rapid disease progression while on first-line TKI therapy who have a very poor prognosis (23-25). There are no prospective randomized trials to address the management of this clinical scenario, and only few retrospective studies focused on this specific issue. However, most of these studies evaluated exclusively the TKI-TKI or TKI-mTOR sequence (24-26). In these and other similar studies, only $40-50 \%$ of patients proceeded from the first line to the second line of treatment. No statistically significant difference was observed in PFS, OS, whether TKI or mTOR was used in the second-line setting $(24,26)$. In the present small cohort of patients, the use of nivolumab after fast progression while on first line therapy resulted in slightly better PFS compared to the remaining patients of the cohort and the ORR was maintained.

The limitations of the present study include a relatively small number of patients and possible patient selection bias favoring long-term responders to previous treatments. There was an imbalance in the number of previous treatment lines between the patients pre-treated with everolimus and others. This may have favored the TKI/mTOR group in terms of OS compared to patients treated with CPI in the second line.

\section{Conclusion}

In conclusion, the results of the present study support the dictum that every patient with mRCC should be treated with CPI provided there are no contraindications (27). Additionally, the present results indicate that patients pretreated with everolimus may also derive benefit from nivolumab and should not be excluded from this therapy. This is especially true for the patients that were pre-treated and responded to multiple therapy lines. The results also lend support to the hypothesis that radiotherapy may potentiate response to subsequent CPI treatment.

\section{Funding}

This work was supported by the Charles University Faculty of Medicine in Hradec Králové grant Progress Q40/06 and Q40/11.

\section{Conflicts of Interest}

$\mathrm{JK}, \mathrm{JKa}, \mathrm{AP}$ and HS received honoraria and travel grants from Novartis, Pfizer, Roche, Bristol Myers Squibb, and Ipsen. BM, TB, $\mathrm{MZ}$ and IK received honoraria and research support from Novartis, Pfizer, Roche, Bristol Myers Squibb, and Ipsen. OK, JH and MS declare no conflicts of interest in relation to this study.

\section{Authors' Contributions}

JK and OK contributed to the study conception and design and they contributed equally to this study. Material preparation, data collection was performed by TB, BM, AP, MZ, JKa, IK, JH, HŠ, MS, JK, OK. Analysis was performed by JK and OK. The first draft of the manuscript was written by $\mathrm{JK}$ and $\mathrm{OK}$ and all authors commented on previous versions of the manuscript. All Authors read and approved the final manuscript.

\section{Acknowledgements}

The Authors would like to thank Bristol Mayers Squibb for enabling early advance access program for our patients with renal cell cancer. The Authors would also like to thank to Dr. Dalibor Svoboda for his help and for performing the statistical analysis.

\section{References}

1 Motzer RJ, Escudier B, McDermott DF, George S, Hammers HJ, Srinivas S, Tykodi SS, Sosman JA, Procopio G, Plimack ER, Castellano D, Choueiri TK, Gurney H, Donskov F, Bono P, Wagstaff J, Gauler TC, Ueda T, Tomita Y, Schutz FA, Kollmannsberger C, Larkin J, Ravaud A, Simon JS, Xu LA, Waxman IM, Sharma $\mathrm{P}$ and CheckMate 025 Investigators: Nivolumab versus everolimus in advanced renal-cell carcinoma. N Engl J Med 373(19): 1803-1813, 2015. PMID: 26406148. DOI: $10.1056 /$ NEJMoa1510665

2 Heng DY, Xie W, Regan MM, Harshman LC, Bjarnason GA, Vaishampayan UN, Mackenzie M, Wood L, Donskov F, Tan MH, Rha SY, Agarwal N, Kollmannsberger C, Rini BI and 
Choueiri TK: External validation and comparison with other models of the International Metastatic Renal-Cell Carcinoma Database Consortium prognostic model: a population-based study. Lancet Oncol 14(2): 141-148, 2013. PMID: 23312463. DOI: $10.1016 / \mathrm{S} 1470-2045(12) 70559-4$

3 Teishima J, Inoue S, Hayashi T, Mita K, Hasegawa Y, Kato M, Kajiwara M, Shigeta M, Maruyama S, Moriyama H, Fujiwara S and Matsubara A: Impact of the systemic immune-inflammation index for the prediction of prognosis and modification of the risk model in patients with metastatic renal cell carcinoma treated with first-line tyrosine kinase inhibitors. Can Urol Assoc J 14(11): E582-E587, 2020. PMID: 32520703. DOI: 10.5489/ cuaj.6413

4 Zhong JH, Huang DH and Chen ZY: Prognostic role of systemic immune-inflammation index in solid tumors: a systematic review and meta-analysis. Oncotarget 8(43): 75381-75388, 2017. PMID: 29088873. DOI: 10.18632 /oncotarget. 18856

$5 \mathrm{Hu} \mathrm{K}$, Lou L, Ye J and Zhang S: Prognostic role of the neutrophil-lymphocyte ratio in renal cell carcinoma: a metaanalysis. BMJ Open 5(4): e006404, 2015. PMID: 25854964. DOI: $10.1136 /$ bmjopen-2014-006404

6 Wang Z, Peng S, Wang A, Xie H, Guo L, Jiang N and Niu Y: Platelet-lymphocyte ratio acts as an independent predictor of prognosis in patients with renal cell carcinoma. Clin Chim Acta 480: 166-172, 2018. PMID: 29462592. DOI: 10.1016/ j.cca.2018.02.014

7 Kopecký J, Kubeček O, Geryk T, Slováčková B, Hoffmann P, Žiaran M and Priester P: Nivolumab induced encephalopathy in a man with metastatic renal cell cancer: a case report. J Med Case Rep 12(1): 262, 2018. PMID: 30217214. DOI: 10.1186/ s13256-018-1786-9

8 Albiges L, Negrier S, Dalban C, Chevreau C, Gravis G, Oudard S, Laguerre B, Barthelemy P, Borchiellini D, Gross-Goupil M, Geoffrois L, Rolland F, Thiery-Vuillemin A, Joly F, Ladoire S, Tantot F, Escudier B and GETUG: Safety and efficacy of nivolumab in metastatic renal cell carcinoma $(\mathrm{mrcc})$ : Final analysis from the nivoren getug afu 26 study. J Clin Oncol 37: 542-542, 2019. DOI: 10.1200/JCO.2019.37.7_suppl.542

9 Albiges L, Negrier S, Dalban C, Gravis G, Chevreau C, Oudard S, Laguerre B, Barthelemy P, Borchiellini D, Gross-Goupil M, Geoffrois L, Brihoum M, Escudier B and GETUG: Safety and efficacy of nivolumab in metastatic renal cell carcinoma (mrcc): Results from the nivoren getug-afu 26 study. J Clin Oncol 36: 577-577, 2018. DOI: 10.1200/JCO.2018.36.6_suppl.577

10 De Giorgi U, Cartenì G, Giannarelli D, Basso U, Galli L, Cortesi E, Caserta C, Pignata S, Sabbatini R, Bearz A, Buti S, Lo Re G, Berruti A, Bracarda S, Cognetti F, Rastelli F, Fornarini G, Porta C, Turci D, Sternberg CN, Procopio G and Italian Nivolumab Renal Cell Cancer Early Access Program Group: Safety and efficacy of nivolumab for metastatic renal cell carcinoma: realworld results from an expanded access programme. BJU Int 123(1): 98-105, 2019. PMID: 29956884. DOI: 10.1111/ bju. 14461

11 Grimm M-O, Grünwald V, Heyde E, Herber M, MuellerHuesmann $\mathrm{H}$ and Bedke $\mathrm{J}$ : Real world data on the use of nivolumab and ipilimumab combination therapy or nivolumab monotherapy in the treatment of renal cell carcinoma: Interim results from the non-interventional study (NIS) NORA. European Urology Open Science 19: e1107, 2020. DOI: $10.1016 /$ S2666-1683(20)33323-1
12 Rini BI, Escudier B, Tomczak P, Kaprin A, Szczylik C, Hutson TE, Michaelson MD, Gorbunova VA, Gore ME, Rusakov IG, Negrier S, Ou YC, Castellano D, Lim HY, Uemura H, Tarazi J, Cella D, Chen C, Rosbrook B, Kim S and Motzer RJ: Comparative effectiveness of axitinib versus sorafenib in advanced renal cell carcinoma (AXIS): a randomised phase 3 trial. Lancet 378(9807): 1931-1939, 2011. PMID: 22056247. DOI: $10.1016 /$ S0140-6736(11)61613-9

13 Huang HF, Fan XR and Ji ZG: The effectiveness of sorafenib over other targeted agents in the second-line treatment of metastatic renal cell carcinoma: a meta-analysis. Pathol Oncol Res 25(4): 1497-1503, 2019. PMID: 30421088. DOI: 10.1007/ s12253-018-0516-3

14 Koutsoukos K, Bamias A, Tzannis K, Espinosa Montaño M, Bozionelou V, Christodoulou C, Stefanou D, Kalofonos H, Duran I and Papazisis K: Real-world experience of everolimus as second-line treatment in metastatic renal cell cancer after failure of pazopanib. Onco Targets Ther 10: 4885-4893, 2017. PMID: 29062235. DOI: 10.2147/OTT.S141260

15 Choueiri TK, Escudier B, Powles T, Tannir NM, Mainwaring PN, Rini BI, Hammers HJ, Donskov F, Roth BJ, Peltola K, Lee JL, Heng DYC, Schmidinger M, Agarwal N, Sternberg CN, McDermott DF, Aftab DT, Hessel C, Scheffold C, Schwab G, Hutson TE, Pal S, Motzer RJ and METEOR investigators: Cabozantinib versus everolimus in advanced renal cell carcinoma (METEOR): final results from a randomised, openlabel, phase 3 trial. Lancet Oncol 17(7): 917-927, 2016. PMID: 27279544. DOI: 10.1016/S1470-2045(16)30107-3

16 Motzer RJ, Hutson TE, Ren M, Dutcus C and Larkin J: Independent assessment of lenvatinib plus everolimus in patients with metastatic renal cell carcinoma. Lancet Oncol 17(1): e4-e5, 2016. PMID: 26758760. DOI: 10.1016/S1470-2045(15)00543-4

17 Huijts CM, Santegoets SJ, de Jong TD, Verheul HM, de Gruijl TD and van der Vliet HJ: Immunological effects of everolimus in patients with metastatic renal cell cancer. Int J Immunopathol Pharmacol 30(4): 341-352, 2017. PMID: 28988508. DOI: $10.1177 / 0394632017734459$

18 Hirayama Y, Gi M, Yamano S, Tachibana H, Okuno T, Tamada S, Nakatani T and Wanibuchi H: Anti-PD-L1 treatment enhances antitumor effect of everolimus in a mouse model of renal cell carcinoma. Cancer Sci 107(12): 1736-1744, 2016. PMID: 27712020. DOI: 10.1111/cas.13099

19 Zhang Q, Liu H, Wang H, Lu M, Miao Y, Ding J, Li H, Gao X, Sun $\mathrm{S}$ and Zheng J: Lenvatinib promotes antitumor immunity by enhancing the tumor infiltration and activation of NK cells. Am J Cancer Res 9(7): 1382-1395, 2019. PMID: 31392076.

20 Boissier R, Campagna J, Branger N, Karsenty G and Lechevallier $\mathrm{E}$ : The prognostic value of the neutrophil-lymphocyte ratio in renal oncology: A review. Urol Oncol 35(4): 135-141, 2017. PMID: 28233671. DOI: 10.1016/j.urolonc.2017.01.016

21 Dalpiaz O, Luef T, Seles M, Stotz M, Stojakovic T, Pummer K, Zigeuner R, Hutterer GC and Pichler M: Critical evaluation of the potential prognostic value of the pretreatment-derived neutrophil-lymphocyte ratio under consideration of C-reactive protein levels in clear cell renal cell carcinoma. Br J Cancer 116(1): 85-90, 2017. PMID: 27907929. DOI: 10.1038/bjc. 2016.393

22 Wang X, Su S and Guo Y: The clinical use of the platelet to lymphocyte ratio and lymphocyte to monocyte ratio as prognostic factors in renal cell carcinoma: a systematic review 
and meta-analysis. Oncotarget 8(48): 84506-84514, 2017. PMID 29137443. DOI: $10.18632 /$ oncotarget 21108

23 Rini BI and Atkins MB: Resistance to targeted therapy in renalcell carcinoma. Lancet Oncol 10(10): 992-1000, 2009. PMID: 19796751. DOI: 10.1016/S1470-2045(09)70240-2

24 Heng DY, Mackenzie MJ, Vaishampayan UN, Bjarnason GA, Knox JJ, Tan MH, Wood L, Wang Y, Kollmannsberger C, North S, Donskov F, Rini BI and Choueiri TK: Primary anti-vascular endothelial growth factor (VEGF)-refractory metastatic renal cell carcinoma: clinical characteristics, risk factors, and subsequent therapy. Ann Oncol 23(6): 1549-1555, 2012. PMID: 22056973. DOI: $10.1093 /$ annonc/mdr533

25 Busch J, Seidel C, Weikert S, Wolff I, Kempkensteffen C, Weinkauf L, Hinz S, Magheli A, Miller K and Grünwald V: Intrinsic resistance to tyrosine kinase inhibitors is associated with poor clinical outcome in metastatic renal cell carcinoma. BMC Cancer 11: 295, 2011. PMID: 21756335. DOI: 10.1186/ 1471-2407-11-295
26 Bersanelli M, Iacovelli R, Buti S, Houede N, Laguerre B, Procopio G, Lheureux S, Fischer R, Negrier S, Ravaud A, Oudard S, Escudier B, Albiges L and Porta C: Metastatic renal cell carcinoma rapidly progressive to sunitinib: What to do next? Eur Urol Oncol 4(2): 274-281, 2021. PMID: 31331862. DOI: 10.1016/j.euo.2019.06.018

27 Sambi M, Bagheri L and Szewczuk MR: Current challenges in cancer immunotherapy: Multimodal approaches to improve efficacy and patient response rates. J Oncol 2019: 4508794, 2019. PMID: 30941175. DOI: 10.1155/2019/4508794

Received June 8, 2021

Revised July 15, 2021

Accepted July 16, 2021 